\title{
Design of Switched-Sector Planar Antenna for Wireless LAN
}

\author{
Hsueh-Jyh Li* ,Chen-Wei Huang \\ Graduate Institute of Communication Engineering, National Taiwan University, Taipei, Taiwan, \\ R.O.C.
}

Abstract

Given the potential market for wireless LAN (WLAN) technology, multiple-sector antenna arrays are known to be able to provide capacity enhancement by means of interference reduction. In this paper, the switched-sector planar antenna will be designed with nine-direction radiation pattern on single PCB board. The kind of antenna can act as an Access Point (AP) in indoor WLAN.

\section{I . INTRODUCTION}

The demand for increased capacity in wireless networks motivated recent research toward wireless systems that exploit space diversity [1]. As a result, there are many efforts on the design of antenna arrays. Research on antenna design has focused on the selection of attractive radiating elements and antenna architecture that can accommodate the physical and electrica requirements of the system [2]. This paper presents a comprehensive effort on antenna-array design. The main goal of this paper is to design switched-sector planar antennas operating at a frequency of $6 \mathrm{GHz}$ and conform to the geometry of an array architecture that could control the radiation pattern both in azimuth and elevation directions. Consequently, this resulted in the selection of microstrip patches arranged in a planar configuration. In addition, the number of radiating elements was chosen to meet bandwidth requirements while maintaining reasonable costs and complexity for hardware implementation.

\section{II . ANTENNA DESIGN}

The type of antenna element considered in this paper is a microstrip antenna (also known as a patch antenna). The microstrip antenna element was designed to operate at a frequency of $6 \mathrm{GHz}(\lambda=5 \mathrm{~cm})$ using a FR4 material with some related parameters shown in Tablel.

\begin{tabular}{|c|c|c|c|c|}
\hline FR4 parameters & $\varepsilon_{r}$ & loss tangent & substrate thickness & metal thickness \\
\hline values & 4.28 & 0.0018 & $1.48 \mathrm{~mm}$ & $0.04 \mathrm{~mm}$ \\
\hline
\end{tabular}

Table l. Parameters of PCB board FR4

According to formulas of patch antenna, the length and width of single patch are $1.21 \mathrm{~cm}$ and $1.92 \mathrm{~cm}$ respectively. The bandwidth efficiency of single patch is $3.17 \%$. Thus, the expected bandwidth of single patch is $190.2 \mathrm{MHz}$.

For an $M \times N$ identical elements with uniform spacings $d x$ and $d y$ placed on the $x y$ - plane, the array factor is given by [3]

$$
[A F(\theta, \phi)]_{M \times N}=\sum_{m=1}^{M} \sum_{n=1}^{N} I_{m n} e^{j k \sin \theta\left[(m-1) d_{x} \cos \phi+(n-1) d_{y} \sin \rho\right]}
$$

where $I_{m n}$ represents the feed current of the $m n$th individual patch elements and $(\theta, \phi)$ represents the elevation and azimuth angles 
The design objective is to establish nine-directional main beams toward different desired directions on a $8 \times 8$ planar microstrip antenna array. The block diagram of the switched-sector planar antenna is shown in Fig1. These antenna elements are spaced at $\lambda / 2$ apart in both directions. Each block uses its own feed network to produce a radiation pattern directed to its desired direction. Once the desired direction of each block is defined, the weighting of each element in $\mathrm{Eq}$ (1) can be determined, and the feed network of each block can then be designed. In this paper, the feed network was designed with uniform amplitude and constant progressive phase shift and it is to be implemented with microstrip lines.

Since the switched-sector antenna array is to be placed on the center ceiling of a large room, the desired pointing direction $\theta_{0}$ and $\phi_{0}$ of each block are designed to be at $\left(\theta_{0}=45^{\circ}, \phi_{0}=45^{\circ}\right),\left(\theta_{0}=135^{\circ}, \phi_{0}=45^{\circ}\right),\left(\theta_{0}=-135^{\circ}, \phi_{0}=45^{\circ}\right),\left(\theta_{0}=-45^{\circ}, \phi_{0}=45^{\circ}\right),\left(\theta_{0}=0^{\circ}, \phi_{0}=\right.$ $\left.45^{\circ}\right),\left(\theta_{0}=180^{\circ}, \phi_{0}=45^{\circ}\right),\left(\theta_{0}=-90^{\circ}, \phi_{0}=45^{\circ}\right),\left(\theta_{0}=90^{\circ}, \phi_{0}=45^{\circ}\right),\left(\theta_{0}=0^{\circ}, \phi_{0}=\right.$ any value $)$ respectively. Substituting these directions to $\mathrm{Eq}(1)$, the required phase of each element can be determined and the feed network of each block can be designed. The result is shown in Fig2. Although there are nine blocks on PCB, it is noted that only four different patterns of feed networks actually need to consider. Blocks $(1,2,3,4)$ have the same antenna array and feed network pattern, but they are oriented with different directions. Blocks $(5,6)$ and Blocks $(7,8)$ are other pairs.

\section{SIMULATION AND MEASUREMENT RESULTS}

The Ensemble simulation tool is used to analyze and simulate the microstrip antenna element and the mutual coupling effects between elements [4]. The software Ensemble is also used to adjust the excitation (magnitudes and phases) network

The PCB layout of the switched-sector planar antenna with radiation patterns of nine directions is shown in Fig2. The measured return losses of different block patterns are shown in Fig3. The respective bandwidths and return losses are shown in Table2.

\begin{tabular}{|c|c|c|c|c|}
\hline Parameters & Right_Top3x3 & Center $2 \times 2$ & Right $2 \times 3$ & Bottom $3 \times 2$ \\
\hline $\mathrm{BW}(\mathrm{MHz})$ & $160 \mathrm{MHz}$ & $160 \mathrm{MHz}$ & $210 \mathrm{MHz}$ & $290 \mathrm{MHz}$ \\
\hline Return $\operatorname{Loss}(\mathrm{dB})$ & $-24.3 \mathrm{~dB}$ & $.14 .9 \mathrm{~dB}$ & $-18.6 \mathrm{~dB}$ & $-37.7 \mathrm{~dB}$ \\
\hline
\end{tabular}

Table2. Measurement results of switched-sector planar array

The measured radiation patterns of different blocks are shown from Fig4 to Fig1l. These figures illustrate measurement results of switched-sector planar antenna at angles $\phi=0^{\circ}$ and $\phi=90^{\circ}$ with both vertical and horizontal polarizations [5]. The individual 3D radiation pattern of each block on switched-sector planar antenna is measured in an anechoic chamber using a Vector Network Analyzer.

\section{CONCLUSIONS}

The design of switched-sector planar antenna with radiation patterns of nine directions has been presented. There is an isolation of at least $15 \mathrm{~dB}$ within the $3 \mathrm{~dB}$ beamwidth between the vertical and horizontal polarization at $\phi=0^{\circ}$ and $\phi=90^{\circ}$. The results obtained in this paper can provide certain guidelines for the measurement of wireless LAN environments.

In the future, this switched-sector antenna will be used to measure the channel characteristics in WLAN environments. It is expected that the multipath delay spread will be reduced if a sector antenna is employed. Consequently, the guard interval required in the OFDM signals can be smaller and the permissible data rate can be increased. 
Acknowledgement: This work was supported by the National Science Council of the Republic of China under the grant number 90-2213-E-002-036 and the MOE program for promoting academic excellence universities under the grant number 89E-FA06-2-4-7.

\section{REFERENCES}

[1] R.H. Roy, "An overview of smart antenna technology: The next wave in wireless communications," in Proc. 1998 IEEE Aerospace Conf., vol.3, May 1998, pp.339-345.

[2] J. Lu and T. Ohira, "Smart antennas at wireless mobile computer terminals and mobile stations," in Proc. 2001 IEEE AP-S Int. Symp. USNC/URSI Nat. Radio Sci. Meeting, Boston, July $8-13,2001$.

[3] Robert S. Elliott, "antenna theory and design," Prentice-Hall, Inc., pp. 197-204.

[4] Bellofiore, S.; Foutz, J.; Govindarajula, R.; Bahceci, I.; Balanis, C.A.; Spanias, A.S. Capone, J.M.; Duman, T.M., "Smart antenna system analysis, integration and performance for mobile ad-hoc networks (MANETs)," IEEE Transactions on Antennas and Propagation, mobile ad-hoc networks (MANETs),

[5] Pesik, L.J; Beach, M.A; Allen, B.H.," Performance analysis of switched-sector antennas for indoor wireless LANs," High Frequency Postgraduate Student Colloquium, 2001, 6th IEEE, 2001 , pp. 30 - 34.

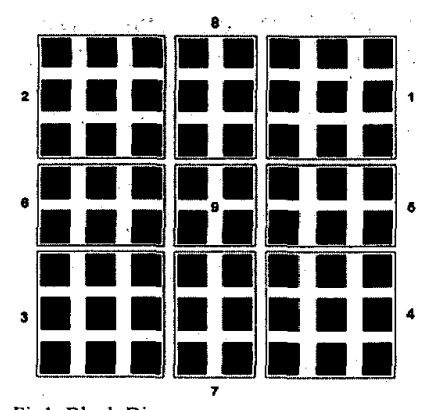

Figl. Block Diagram

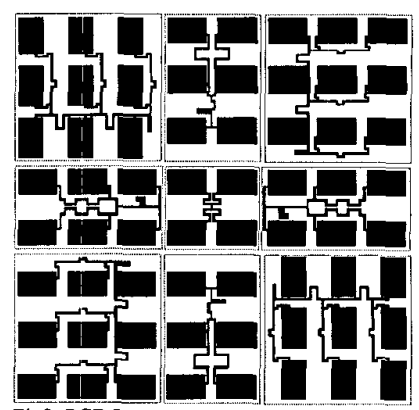

Fig2. PCB Layout

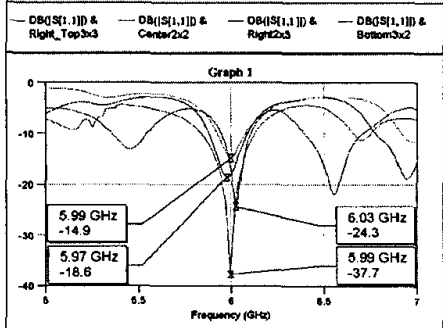

Fig3. Measurement results of Return Loss 


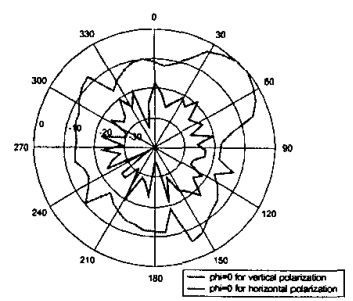

Fig4. Radiation pattern of Block 1 at $P h i=0^{\circ}$

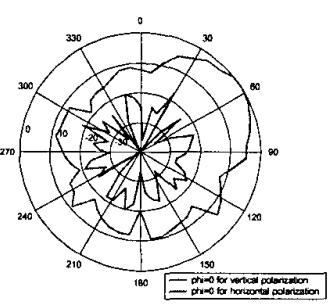

Fig6. Radiation pattern of Block5 at $\mathrm{Phi}=0^{\circ}$

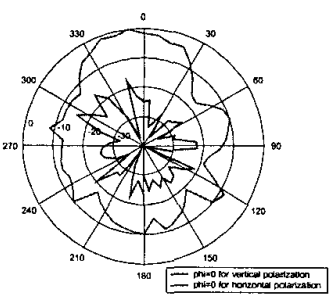

Fig8. Radiation pattern of $\mathrm{Block} 7$ at $\mathrm{Phi}=0^{\circ}$

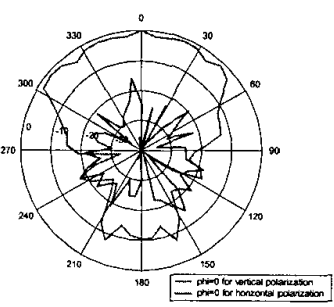

Fig 10. Radiation pattern of Block9 at $\mathrm{Phi}=\mathbf{0}^{\circ}$

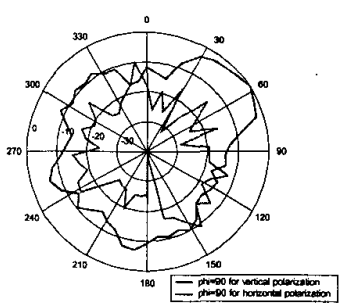

Fig5. Radiation pattern of Block1 at $\mathrm{Phi}=90^{\circ}$

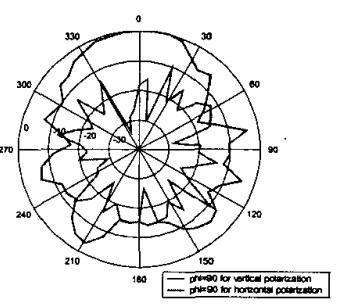

Fig7. Radiation pattern of Block5 at $\mathrm{Phi}=90^{\circ}$

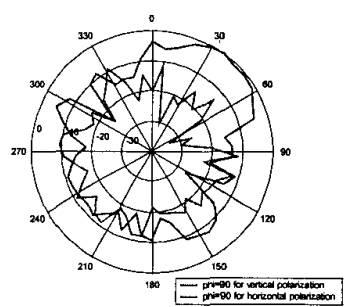

Fig9. Radiation pattern of Block7 at Phi $=90^{\circ}$

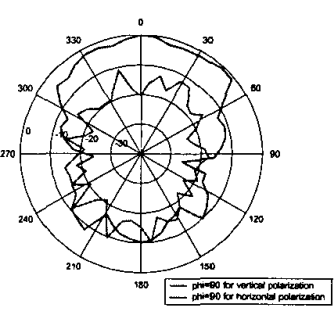

Fig11. Radiation pattern of Block9 at $\mathrm{Phi}=90^{\circ}$ 